\title{
Parasite Immunology
}

\section{Volume 43, Issue 4, April 2021, Article number e12814}

\section{Evolution of antigen-specific immune responses in cutaneous leishmaniasis patients}

\author{
Akram Miramin Mohammadi $^{\mathrm{a}}$, Malcolm S. Duthie ${ }^{\mathrm{b}}$, Steven G Reed ${ }^{\mathrm{b}}$, Amir Javadi ${ }^{\mathrm{c}}$, Ali \\ Khamesipour $^{\mathrm{a}}$ \\ ${ }^{a}$ Center for Research \& Training in Skin Diseases \& Leprosy (CRTSDL), Tehran University of \\ Medical Sciences (TUMS), Tehran, Iran \\ ${ }^{\mathrm{b}}$ HDT Bio, Seattle, WA, Saudi Arabia \\ ${ }^{\mathrm{c}}$ Department of Social Medicines, Qazvin University of Medical Sciences, Qazvin, Iran \\ Corresponding author: Khamesipour, A.; Center for Research \& Training in Skin Diseases \& \\ Leprosy (CRTSDL), Tehran University of Medical Sciences (TUMS), Tehran, Iran; \\ email:ali.khamesipour@gmail.com
}

Abstract

Aims: Despite immunization appearing to be the most appropriate strategy for long-term control of the vector-borne leishmaniases, no sustainable vaccine is currently available against any form of leishmaniasis. We therefore evaluated, in the context of vaccine antigen candidates, antigenspecific immune response at various stages of cutaneous leishmaniasis (CL). Methods and results: Peripheral blood mononuclear cells (PBMC) isolated from healthy volunteers and CL patients (caused by either Leishmania major or L tropica) were incubated with crude Leishmania proteins (soluble Leishmania antigen; SLA), single recombinant proteins (TSA, LeIF, LmSTI1) or chimeric fusion proteins (LEISH-F2 and LEISH-F3). The concentrations of immune modulatory cytokines were then determined. While we did not detect appreciable antigen-specific IL-5 secretion, SLA induced secretion of interleukin (IL)-10 in cultures from early active lesion CL patients and even from healthy individuals. Conversely, interferon (IFN)- $\gamma$ responses to SLA and recombinant proteins followed a similar pattern, developing only in the late active CL lesion phase. Once established, antigen-specific IFN- $\gamma$ responses persisted in cured CL patients. Conclusion: Together, our results provide further insight into the development of immune responses during CL and further validate the selection of LEISH-F2 and LEISH-F3 as vaccine antigen candidates. 